\section{Hepatite por Propiltiouracil: Apresentação de Dois Casos com Evoluções Diversas}

\section{RESUMO}

Os autores apresentam dois casos de hepatite tóxica por uso de propiltiouracil (PTU), com evoluções diversas. Uma paciente desenvolveu ictericia e alterações de transaminases, que desapareceram com a suspensão do medicamento. Outra paciente apresentou-se com quadro de hemorragia digestiva alta, insuficiência hepática e evoluiu rapidamente para o óbito. Os dois casos mostraram sorologias negativas para hepatites virais. Os autores chamam atenção para a importância da orientação ao paciente quanto à possibilidade de desenvolver icterícia em uso de PTU e, nos casos de hepatotoxicidade, da pronta suspensão do uso da droga e acompanhamento clínico do paciente. (Arq Bras Endocrinol Metab 1999;43/2: 129-133)

Unitermos: Hepatite; Hepatotoxicidade; Propiltiouracil; Drogas antitireoideanas

\begin{abstract}
The authors present two cases of PTU-associated hepatotoxicity which had different outcome. One patient developed jaundice and altered transaminases, that disappeared after the discontinuation of PTU. The other patient presented with upper gastrointestinal bleeding, hepatic failure and died soon after the admission. Laboratory evaluation showed negative sorology for viral hepatitis in both cases. The authors highlight the importance of the orientation of the patient about the possibility of developing jaundice during the use of PTU and in cases of hepatotoxicity they orient the immediate withdraw of the drug and clinical follow-up.
\end{abstract} (Arq Bras Endocrinol Metab 1999;43/2: 129-133)

Keywords: Hepatitis; Hepatotoxicity; Propylthiouracil; Anti-thyroid drugs

O Propiltiouracil (PTU) assumiu um importante papel no tratamento do hipertireoidismo em todo o mundo, desde sua introdução em 1946 (1). Os efeitos colaterais mais freqüentes das drogas anti-tireoideanas são "rash" cutâneo, urticária, artralgia, febre e leucopenia transitória, que estima-se estarem presentes $\mathrm{cm} 1$ a $5 \%$ dos casos - sendo considerados efeitos adversos de menor importância. Dentre os efeitos colaterais potencialmente graves, a agranulocitose é o mais comum e temido, acometendo 0,2 a $0,5 \%$ dos pacientes, tanto devido ao PTU quanto ao metimazol (2). Entretanto, apesar de raras, têm aumentado as descrições de hepatotoxicidade destas drogas, particularmente a hepatite tóxica pelo PTU (3-17). Quando esta complicação é precocemente reconhecida e o tratamento com a droga é suspenso, seu curso clínico geralmente é benigno. Todavia, têm sido relatados diversos casos de óbito por insuficiência hepática decorrentes do tratamento com PTU $(5,9,11,14,16)$. Apesar de ser evento raro, a hepatite pelo PTU exige atenção imediata da equipe médica, que deve suspender o medicamento e acompanhar o caso com avaliações freqüentes de função hepática. Neste trabalho, descrevemos dois casos com evoluções distintas desta complicação, discutindo a importância do pronto reconhecimento e atendimento ao paciente acometido.

\section{apresentação de caso}

Departamento de Clínica Médica da Universidade Federal de Uberlândia, $M G$

\author{
João C. Gonçalves Júnior \\ Rodrigo de A. Oliveiva \\ Ademar M. de Carvalho \\ Paulo Tannús Jorge
}




\section{Caso 1}

Uma mulher de 34 anos, descendente de asiáticos, apresentou-se em Julho de 1996 com quadro clínico de tireotoxicose, bócio difuso e ausência de alterações oculares. Negou uso de qualquer medicamento. A história familiar foi negativa para patologias tireoideanas ou hepáticas. À avaliação laboratorial mostrou TSH indetectável (V.R.: 0,3 a $4,0 \mathrm{mU} / \mathrm{L}$ ), FT4: $6,9 \mathrm{ng} / \mathrm{ml}$ (V.R.: 0,75-1,8 ng/ml), T3: 5,24 ng/ml (V.R.: 0,7$2,0 \mathrm{ng} / \mathrm{ml}$ ), anticorpo anti-microssomal positivo em título de $1 / 25.600$, e soro não reativo para anticorpo anti-tireoglobulina. O hemograma apresentou-se normal (6.700 leucócitos com $47 \%$ de neutrófilos). Foi iniciado tratamento com propranolol $40 \mathrm{mg}$ por dia e propiltiouracil $600 \mathrm{mg}$ por dia.

Três semanas após introdução do PTU retornou com hemograma normal, TSH: 0,002 $\mathrm{mU} / \mathrm{L}, \mathrm{T} 3: 1,77 \mathrm{ng} / \mathrm{ml}$ e T4: $13,0 \mu \mathrm{g} / \mathrm{dl}$ (V.R.: 4,5$12,5 \mu \mathrm{g} / \mathrm{dl}$ ), e referindo icterícia, urina escura e fezes claras. Suspendeu-se o uso da droga anti-tireoideana, e os exames da época mostraram os seguintes resultados: Aspartato aminotransferase (AST): 87,0 U/L (V.R.: até $3 \mathrm{l} \mathrm{U} / \mathrm{L}$ ); Alanina aminotransferase (ALT): 173,0 U/L (V.R.: até $31 \mathrm{U} / \mathrm{L}$ ); Fosfatase Alcalina: $333 \mathrm{U} / \mathrm{L}$ (V.R.: 42 a $98 \mathrm{U} / \mathrm{L}$ ); gama-glutamil transpeptidase (gama-GT): 164,0 U/L (V.R.: 7 a $32 \mathrm{U} / \mathrm{L}$ ); Bilirrubinas Totais: $2,31 \mathrm{mg} / \mathrm{dl}$ (V.R.: até $1,0 \mathrm{mg} / \mathrm{dl}$ ); Bilirrubina Direta: $1,07 \mathrm{mg} / \mathrm{dl}$ (V.R.: até $0,2 \mathrm{mg} / \mathrm{dl}$ ) e Indireta: $1,24 \mathrm{mg} / \mathrm{dl}$ (V.R.: até $0,8 \mathrm{mg} / \mathrm{dl}$ ); anticorpos anti-Vírus $\mathrm{C}$ foram negativos (tanto anti-core quanto anti-proteínas não-estruturais), Antígeno de superficie do Vírus B (HbsAg) negativo e Fator Antinuclear negativo. Duas semanas após a suspensão da droga, manteve-se em tireotoxicose, anictérica e em uso de propranolol $60 \mathrm{mg}$ por dia, sendo encaminhada para tratamento com iodo radioativo.

Cinco semanas após dose de $555 \mathrm{MBq}$ (15 $\mathrm{mCi}$ ) de iodo radioativo, apresentou aminotransferases e bilirrubinas normais, TSH indetectável, T3: 2,47 $\mathrm{ng} / \mathrm{ml} ; \mathrm{T} 4: 2 \mathrm{l}, 9 \mu \mathrm{g} / \mathrm{dl}$. Dois meses após o tratamento ablativo foi suspenso o propranolol e iniciou levotiroxina sódica para tratamento de hipotireoidismo.

\section{Caso 2}

Trata-se de uma paciente de 23 anos, que estava em uso regular de propranolol e PTU há $\mathrm{l}$ ano, em doses desconhecidas, para tratamento de hipertireoidismo. Apresentava icterícia progressiva, iniciada há cerca de 90 dias, e evoluía com hemorragia digestiva alta, de sete dias de duração, quando procurou o serviço de emergência. À admissão apresentava-se comatosa, com icterícia importante, exoftalmia bilateral, hipotensa, apresentando hepatomegalia dolorosa, sem estigmas de doença hepática crônica.

Os exames complementares revelavam ALT: $191 \mathrm{U} / \mathrm{L}$, AST: $513 \mathrm{U} / \mathrm{L}$, Fosfatase Alcalina e gamaGT normais, porém com hiperbilirrubinemia (Bilirrubina Total $=28,24 \mathrm{mg} / \mathrm{dl}$ ), predominando Bilirrubina Direta $(16,46 \mathrm{mg} / \mathrm{dl})$. A Atividade de Protrombina era de $3 \%$, e o hemograma evidenciava anemia normocítica normocrômica ( $\mathrm{Hb}=8,9 \mathrm{~g} \%$ ), com 18.200 leucócitos, sendo $1 \%$ de bastonetes, $41 \%$ de segmentados, $30 \%$ de monócitos e importante cosinofilia de $28 \%$. As sorologias eram negativas para Vírus das Hepatites A (anti-HAV IgM - ELISA), B (HBsAg e anti-HBc IgM - ELISA) e C (anti-HCV - ELISA).

Esta paciente evoluiu rapidamente para óbito, apesar do tratamento com plasma fresco congelado, vitamina $\mathrm{K}$, concentrado de hemáceas e medidas para encefalopatia hepática. A necrópsia parcial revelou broncopneumonia, não definiu etiologia da hemorragia digestiva alta, e, ao exame histológico do figado, detectaram-se extensas áreas de necrose hepática, com proliferação de canalículos biliares e infiltrado mononuclear e eosinofílico multifocal, compatível com Necrose Hepática Maciça.

\section{DISCUSSĀO}

A lesão hepática induzida por PTU é considerada por Cooper (2) como complicação muito rara deste tratamento, ocorrendo em menos de $0,2 \%$ dos casos, apesar de haver dificuldade em se estabelecer com precisão este dado, como visto em estudo de coorte por Liaw e colaboradores em 1993 (18).

As primeiras descrições de hepatite por droga anti-tireoideana foram publicadas mais de 2 anos após o início de seu uso em 1943. Grainger e colaboradores (19), em 1945, não comentam sobre qualquer alteração hepática ao discutir os efeitos tóxicos do tiouracil, enquanto Gargill e colaboradores (20), no mesmo ano, descrevem um caso de icterícia com achado de estase biliar aguda, ocorrido após o uso do tiouracil. Em 1946, Moore e colaboradores (21), em um estudo cooperativo multicêntrico visando descrever as manifestações tóxicas desta droga, cita de modo breve que já havia sido relatado dano hepático grave, mas que as lesões não se mostraram fatais. No mesmo ano Astwood \& VanderLaan (22) publicam o primeiro estudo avaliando exclusivamente o uso de propiltiouracil e, dentre os 100 casos, houve um relato de óbito por insuficiência hepática durante o uso da 
droga, porém, em paciente sabidamente com cirrose avançada prévia. A primeira evidência de hepatotoxicidade pelo PTU é de Livingston \& Livingston em 1947 (3), ao que se seguiram várias outras descrições até a atualidade (3-17). Salientamos que à época destes relatos preliminares não haviam critérios estabelecidos ou propostos para relacionar a disfunção hepática à droga, o que era feito através da relação temporal, e da exclusão de outras etiologias, com os poucos recursos disponíveis então, o que permite questionar algum eventual erro diagnóstico.

Até 1953, quando Eisen (5) descreveu um caso de óbito por hepatite fulminante pelo PTU, năo havia se discutido sobre o diagnóstico diferencial entre o impacto do hipertireoidismo sobre a função hepática e a hepatotoxicidade do PTU. Contudo, Rezek (23), em 1947, relatou que o tiouracil isoladamente não tinha comprovada hepatotoxicidade, e que um prévio dano hepático devido à própria tireotoxicose seria um fator de risco. Atualmente não há evidências convincentes na literatura de que a doença tireoideana cause hepatopatia significativa, na ausência de insuficiência cardíaca congestiva; entretanto, sabe-se que pode piorar os efeitos de outras doenças que afetam o figado $(6,24)$.

A apresentação clínica dos casos de hepatotoxicidade pelo PTU é inespecífica. Varia de quadros assintomáticos, apresentando apenas anormalidade ou piora dos testes bioquímicos (18), até a insuficiência hepática terminal $(5,9-11,14,16)$. Na grande maioria dos casos, o padrão bioquímico é de necrose hepatocelular, com predominante aumento de aminotransferases. Apesar de classicamente relacionada ao metimazol e carbimazol (25), a colestase já foi atribuída ao PTU em pelo menos um relato (26); e por outro lado, há um relato de disfunção hepática com marcada elevação de transaminases e evidência histológica de hepatite focal, devida ao metimazol (27). Como não dispomos de estudo histopatológico do "caso l", não podemos distinguir entre uma lesão hepatocelular discreta e uma colestase simples.

A maior parte dos eventos tóxicos ocorre de $\mathrm{l}$ a 3 meses após o início da terapia $(2,28)$, mas pelo menos um caso já foi descrito com manifestações hepáticas após mais de um ano de uso da droga (11), o que é compatível com o "caso 2" deste trabalho.

A existência de disfunção hepática prévia ao início da droga, devido ao próprio hipertireoidismo, não parece influenciar o surgimento ou gravidade do quadro tóxico $(2,10)$. A reintrodução do medicamento, após suspensão por qualquer motivo, tem sido implicada em maior incidência de hepatotoxicidade
$(2,29)$. Em trabalho de Werner e colaboradores, em 1989 (30), o dano hepático por PTU ocorreu em 4 de 92 pacientes no grupo tratado com altas doses da droga (média de $728 \mathrm{mg}$ por dia) e em nenhum dos 39 pacientes tratados com baixas doses (média de 255 $\mathrm{mg}$ por dia). Contudo, não está determinado que a hepatotoxicidade pelo PTU seja dose-dependente. $(10,14-17)$

A biópsia hepática, em geral, mostra necrose hepatocelular inespecífica, variando na intensidade conforme a gravidade do quadro clínico $(5-8,10,11$, $13,14,16,18,25)$. Os casos de óbito em insuficiência hepática apresentavam necrose maciça ou submaciça do figado, como no caso 2 deste relato. Chama a atenção ainda, nesta paciente, o importante infiltrado eosinofilico ao exame microscópico do figado; isto é descrito como presente, mas não intenso, em vários trabalhos $(6-8,10,11)$. Nos 3 casos biopsiados do trabalho de Liaw e colaboradores (18), nos quais a disfunção hepática foi assintomática, houve citação explícita que eosinófilos não foram encontrados. Vale ressaltar ainda que o hemograma de nossa paciente (caso 2) apresentava eosinofilia importante, lembrando reação de hipersensibilidade, o que não é citado nos demais relatos $(5,6,8,9,11,15,16)$; todavia, temos de enfatizar que esta é uma alteração bastante inespecífica, e outras causas de eosinofilia não puderam ser afastadas, devido à natureza fulminante do quadro hepático.

O mecanismo da hepatotoxicidade ao PTU é desconhecido, embora estudos de sensibilização de linfócitos tenham sugerido uma reação imune à droga $(8,12,17)$.

O diagnóstico de hepatite tóxica por droga antitireoideana, em algumas situações, permanece obscuro. Na literatura há tentativas de se obter meios de confirmar a relação droga-hepatotoxicidade, como estudos de sensibilização de linfócitos $(7,8,12,17,31)$, estudos histológicos $(9,31)$ e novo desafio com a droga $(13,23,31)$, a maior parte sendo clinicamente de pouca aplicabilidade ou até eticamente inaceitáveis em certas condições. Objetivando clarear esta questão, Hanson (9) propõe os seguintes critérios práticos: 1) evidência clínica e laboratorial de disfunção hepatocelular; 2) início dos sintomas serem temporalmente relacionados à terapia com droga; 3) nenhuma evidência sorológica de infecções por vírus da hepatite A, B, Citomegalovírus ou Epstein-Barr Vírus; 4) ausência de condições clínicas sistêmicas lesivas ao figado, como choque ou sepse; 5) nenhuma evidência de doença hepática crônica; e 6) ausência de outras drogas administradas concomitantemente, com potencial hepatotóxico. Hoje, poderíamos acrescentar a sorologia 
negativa para vírus $\mathrm{C}$ como parte do terceiro critério. Nossos casos apresentaram disfunção hepática em uso de PTU e propranolol, sem evidências de outras etiologias. O propranolol não é relacionado a hepatotoxicidade significativa (32) e, no caso l deste relato, esta droga foi mantida continuamente, inclusive durante o período de normalização das provas hepáticas.

Em caso de disfunção hepática clinicamente evidente, é indicada a pronta suspensão do uso do PTU, podendo ser mantido o beta-bloqueador se necessário. Contudo, a literatura não é clara se a rápida interrupção do tratamento garante uma boa evolução do quadro hepático. Ao contrário, há casos de evolução para insuficiência hepática terminal, independente da conduta ágil e adequada $(9-11,14,16)$. De modo geral, comenta-se que a interrupção do tratamento logo aos primeiros sinais e sintomas de hepatite conduz à recuperação progressiva das provas hepáticas na maioria dos casos $(9,10)$.

Por razões éticas óbvias, não existem trabalhos citando experiência com a manutenção do PTU em casos de hepatite clinicamente manifesta. $\mathrm{O}$ caso 2 do presente relato, em que a paciente manteve o uso da droga inadvertidamente, apesar do quadro de hepatite, e sem supervisão médica no período, ilustra uma má evolução com a manutenção do tratamento. Entretanto, é impossivel assegurar que a evolução não teria sido a mesma com a pronta interrupção do PTU.

O estudo de Liaw e colaboradores (18), no qual se detectou $28 \%$ de alterações das transaminases durante o uso do PTU, sem clínica de hepatite, concluiu que a terapia pode ser mantida com cautela na ausência de sintomas e hiperbilirrubinemia, visto que as alterações foram em geral leves e transitórias.

Em conclusão, as recomendações gerais para o manejo da hepatotoxicidade pelo PTU (10) incluem pronta suspensão da droga em caso de significantes anormalidades de enzimas hepáticas e pesquisa de outras causas de hepatopatia; seguimento clínico intensivo, já que a disfunção pode progredir mesmo com a suspensão da droga; reconhecimento precoce da necessidade, e encaminhamento para transplante hepático quando indicado; avaliar a função tireoideana com T4 livre e não T4 total, que é adversamente afetado pela hiperbilirrubinemia; pronto tratamento da doença tireoideana subjacente, para que o hipertireoidismo persistente não contribua com a deterioração ciinica; não reintroduzir o PTU mesmo após normalização da disfunção hepática, devido à sua possível natureza auto-imune.

\section{REFERÊNCIAS}

1. Cooper DS. Which anti-thyroid drug? Am J Med 1986:80:1165-8.

2. Cooper DS. Treatment of Thyrotoxicosis. In: Braverman LE, Utiger RD (eds). Werner and Ingbar's The Thyroid. Philadelphia: JB Lippincott, $7^{\text {th }}$ Ed, 1996:713-34.

3. Livingston HJ, Livingston SF. Agranulocytosis and hepatocellular jaundice. JAMA 1947;135:422-5.

4. Colwell Jr AR, Sando DE, Lang SJ. Propylthiouracilinduced agranulocytosis, toxic hepatitis, and death: report of a case. JAMA 1952;148:639-41.

5. Eisen MJ. Fulminant hepatitis during treatment with propylthiouracil. N Engl J Med 1953;249:814-6.

6. Fedotin MS, Lefer LG. Liver disease caused by propylthiouracil. Arch Intern Med 1975; 135:319-21.

7. Parker $L N$, Hepatitis and propylthiouracil. Ann Intern Med 1975;82:228-9

8. Mihas AA, Holley P, Koff RS, Hirschowitz BI. Fulminant hepatitis and lymphocyte sensitization due to propylthiouracil. Gastroenterology 1976;70:770-4.

9. Hanson JS. Propylthiouracil and hepatitis: two cases and a review of the literature. Arch Intern Med 1984; 144:994-6.

10. Williams KV, Nayak S, Becker D. Reyes J, Burmeister LA Fiffy years of experience with propylthiouracil-associated hepatotoxicity: what have we learned? J Clin Endocrinol Metab 1997;82:1727-33.

11. Limaye A. Ruffolo PR. Propylthiouracil-induced fatal hepatic necrosis. Am J Gastroenterol 1987;82:152-4.

12. Bloch CA, Jenski LJ, Balistreri WF, Dolan LM. Propylthiouracil-associated hepatitis. Arch Intern Med $1985 ; 145: 2129-30$.

13. Weiss $M$. Hassin D, Bank $H$. Propylthiouracil-induced hepatic damage. Arch Intern Med 1980; 140:1 184-5.

14. Safani MM, Tatro DS, Rudd P. Fatal propylthiouracilinduced hepatitis. Arch Intern Med 1982;142:838-9.

15. Westphal S A. Hepatotoxicity from propylthiouracil. South Med J 1994;87:943-7.

16. Jonas MM, Eidson MS. Propylthiouracil hepatotoxicity: two pediatric cases and review of the literature. J Pediatr Gastroenterol Nutr 1988;7:776-9.

17. Parker WA. Propylthiouracil-induced hepatotoxicity. Clin Pharm 1982;1:471-4.

18. Liaw YF, Huang MJ, Fan KD, Li KL, Wu SS, Chen TJ. Hepatic injury during propylthiouracil therapy in patients with hyperthyroidism: a cohort study. Ann Intern Med 1993; 118:424-8.

19. Grainger A, Gregson DA, Pemberton HS. Thiouracil in the treatment of thyrotoxicosis. BMJ 1945;2:343-5.

20. Gargill SL, Lesses MF. Toxic reactions to thiouracil: report of cases with one fatality. JAMA 1945;127:890-8.

21. Moore D. Toxic manifestations of thiouracil therapy. JAMA 1946; 130:315-9.

22. Astwood EB, VanderLaan WP. Treatment of hyperthyroidism with propylthiouracil. Ann Intern Med $1946 ; 25: 813-21$. 
23. Rezek PR. Relation between Graves' Disease and liver pathology: importance in the use of thiouracil. South Med J 1947;40:166-71.

24. Sellin RV, Sellin JH. The Gastrointestinal Tract and Liver in Thyrotoxicosis. In: Braverman LE, Utiger RD (eds). Werner and Ingbar's The Thyroid. Philadelphia: JB Lippincolt, $7^{\text {th }}$ Ed, 1996:632-6.

25. Vitug AC, Goldman JM. Hepatotoxicity from antithyroid drugs. Horm Res 1985;21:229-34.

26. Seidman DS, Livni E, llie B, Blum I. Propylthiouracil-induced cholestatic jaundice. Clin Toxicol 1986;24:353-60.

27. Becker CE, Gorden P, Robbins J. Hepatitis from methimazole during adrenal steroid therapy for malignant exophtalmos. JAMA 1968;206: 1787-9.

28. Cooper DS. Anti-thyroid drugs. N Engl J Med 1984;311:1353-62.

29. Pimentel Filho FR, Goldman J, Liberman B. Terapêutica descontínua como fator precipitante de reações adversas ao propiltiouracil. Arq Bras Endocrinol Metab 1994;38:130-4.
30. Werner MC, Romaldini JH, Bromberg N, Werner RS, Farah CS. Adverse effects related to thionamide drugs and their dose regimen. Am J Med Sci 1989;297:216-9.

31. Blom H, Stolk J, Schreuder HB, von Blomberg-van der Flier M. A case of carbimazole-induced intrahepatic cholestasis: an immune-mediated reaction? Arch Intern Med 1985; 145:1513-5.

32. Stephen S A. Unwanted effects of propranolol. Am J Cardiol 1966; 18:463-72.

\section{Endereço para correspondência:}

Paulo Tannús Jorge

Rua Geraldo Morais 1115 , ap. 403

38400-020 Uberlândia, $M G$ 